\title{
Separability and entanglement in finite dimer-type chains in general transverse fields
}

\author{
N. Canosa, R. Rossignoli, and J. M. Matera \\ Departamento de Física-IFLP, Universidad Nacional de La Plata, C.C. 67, La Plata 1900, Argentina \\ (Received 20 July 2009; revised manuscript received 23 November 2009; published 11 February 2010)
}

\begin{abstract}
We determine the conditions under which general dimer-type spin chains with $X Y Z$ couplings of arbitrary range in a general transverse field will exhibit an exactly separable parity-breaking eigenstate. We also provide sufficient conditions which ensure that it will be a ground state. We then examine the exact side limits at separability of the entanglement between any two spins in a finite chain, showing that in the vicinity of separability, the system will loose all signatures of dimerization, with pairwise entanglement approaching infinite range and becoming independent of separation and interaction range. The possibility of a nonuniform exactly separable ground state induced by an alternating field is also shown. As illustration, we examine the behavior of the pairwise entanglement in a finite $X Y$ dimer chain under a uniform as well as alternating field. Related aspects of the magnetization are also discussed.
\end{abstract}

DOI: 10.1103/PhysRevB.81.054415

PACS number(s): 03.67.Mn, 03.65.Ud, 75.10.Jm, 64.70.Tg

\section{INTRODUCTION}

Quantum entanglement is an essential resource for quantum information science, allowing radically new forms of information transmission and processing, ${ }^{1-4}$ It has also aroused great interest in condensed matter and many-body physics, ${ }^{5}$ providing a different perspective for the analysis of strongly correlated systems. Fundamental properties of entanglement in quantum spin chains have been determined, especially in connection with critical phenomena in the thermodynamic limit. ${ }^{5-7}$ The study of finite spin chains can also provide insights into the most basic aspects of entanglement and is presently also stimulated by the unprecedented level of control that can be reached in some recently developed quantum devices, ${ }^{8,9}$ able to realize spin arrays with controllable Heisenberg interactions.

A fundamental related question is the range the entanglement between individual spins can reach under the action of an applied magnetic field. At the standard critical field of large anisotropic $X Y$ or $X Y Z$ chains, it remains finite and typically small (for instance, restricted to just first and second neighbors in a $1 D$ Ising chain in a transverse field) ${ }^{6}$ However, it can diverge at a different field: anisotropic chains may also exhibit a factorizing field, where an exactly separable ground state (GS) becomes possible, i.e., where the mean field GS becomes exact. This remarkable feature was first discovered in $1 D$ chains with first-neighbor couplings ${ }^{10,11}$ and recently examined in detail in more general systems in a uniform field. ${ }^{12-19}$ A general method for determining separability was in particular developed in Refs. 17 and 18. In the immediate vicinity of the factorizing field, the pairwise entanglement in a finite chain can reach full range. ${ }^{14,16}$ The transverse factorizing field in finite $X Y Z$ chains arises actually at the crossing of opposite $S_{z}$-parity levels ${ }^{16}$ with separable parity-breaking eigenstates emerging from the superposition of the entangled definite parity states. $^{20}$

The aim of this work is to examine the previous issues in finite dimer-type arrays, which have recently received much attention. ${ }^{17,19,21,22}$ We will consider arrays of arbitrary spins with $X Y Z$ couplings of arbitrary range in a transverse field, not necessarily uniform, and determine the separability conditions together with the entanglement side limits at separability, which will be shown to be independent of separation, coupling range and other details such as the strength of the coupling between dimers. At these points all traces of dimerization will then be lost. We will also examine factorization under an alternating field, which can give rise to a separability curve with field dependent separable solutions and entanglement limits. Entanglement between spins unconnected by the interaction can in this way exceed that between linked pairs in the vicinity of separability. These effects are especially noticeable for finite chains close to the $X X Z$ limit. Related aspects of the magnetization and the entanglement between one spin and the rest of the chain are also discussed.

Section II contains the general theoretical results, including the mean field+RPA interpretation of the separability conditions, while Sec. III the application to general dimertype chains, including illustrative exact results for finite chains. The appendix contains the details of the exact calculation obtained through the Jordan-Wigner transformation. Conclusions are finally drawn in Sec. IV.

\section{FORMALISM}

\section{A. Transverse factorizing fields}

We first consider the general Hamiltonian

$$
H=\sum_{i} b^{i} s_{i}^{z}-\frac{1}{2} \sum_{i \neq j}\left(v_{x}^{i j} s_{i}^{x} s_{j}^{x}+v_{y}^{i j} s_{i}^{y} s_{j}^{y}+v_{z}^{i j} s_{i}^{z} s_{j}^{z}\right)
$$

which describes an array of $n$ spins $s_{i}$ not necessarily equal, interacting through $X Y Z$-type couplings of arbitrary range in a general transverse applied field $b^{i}$. It satisfies $\left[H, P_{z}\right]=0$, where $P_{z}=\exp \left[i \pi \sum_{i=1}^{n}\left(s_{i}^{z}+s_{i}\right)\right]$ denotes the global $S_{z}$ parity or phase flip (here $s_{i}$ is the spin value at site $i$ ). Denoting with $\left|0_{i}\right\rangle$ the local state with its spin fully aligned along the $-z$ direction $\left(s_{i}^{z}\left|0_{i}\right\rangle=-s_{i}\left|0_{i}\right\rangle\right)$, this Hamiltonian will exhibit a fully separable parity-breaking eigenstate of the form 


$$
|\Theta\rangle \equiv\left|\theta_{1} \ldots \theta_{n}\right\rangle=\otimes_{i=1}^{n} \exp \left[i \theta_{i} s_{i}^{y}\right]\left|0_{i}\right\rangle,
$$

i.e., a state with its spins fully aligned along local axes forming angles $\theta_{i}$ with the $z$ axis, if (and only if) the conditions

$$
\begin{gathered}
v_{y}^{i j}=v_{x}^{i j} \cos \theta_{i} \cos \theta_{j}+v_{z}^{i j} \sin \theta_{i} \sin \theta_{j}, \\
b^{i} \sin \theta_{i}=\sum_{j \neq i} s_{j}\left(v_{x}^{i j} \cos \theta_{i} \sin \theta_{j}-v_{z}^{i j} \sin \theta_{i} \cos \theta_{j}\right),
\end{gathered}
$$

are satisfied. ${ }^{20}$ They can be obtained replacing $s_{i}^{\mu}$ in $H$ by the rotated operators $e^{-i \theta_{i} s_{i}^{y}} S_{i}^{\mu} e^{i \theta_{i} s_{i}^{y}}$ and solving $H_{\Theta}|0\rangle=E_{\theta}|0\rangle$, where $H_{\Theta}=e^{-i \Sigma_{i} \theta_{i} s_{i}^{y}} H e^{i \Sigma_{i} \theta_{i} s_{i}^{y}}$ and $|0\rangle=\otimes_{i}\left|0_{i}\right\rangle$. Equations (3) and (4) actually hold for general local rotations $e^{i \phi_{i} s_{i}}\left|0_{i}\right\rangle$ since the latter can also be cast in the form (2) through complex angles $\theta_{i}$ and a suitable normalization factor. ${ }^{20,23}$ Note also that for a spin $1 / 2$ array Eq. (2) is in fact the most general separable state. The energy $E_{\Theta}$ becomes

$$
\begin{aligned}
E_{\Theta}= & -\sum_{i=1}^{n} s_{i}\left[b^{i} \cos \theta_{i}+\frac{1}{2} \sum_{j \neq i} s_{j}\left(v_{x}^{i j} \sin \theta_{i} \sin \theta_{j}\right.\right. \\
& \left.\left.+v_{z}^{i j} \cos \theta_{i} \cos \theta_{j}\right)\right] .
\end{aligned}
$$

If $\left|v_{y}^{i j}\right| \leq v_{x}^{i j} \forall i, j$ and $\theta_{i} \in(0, \pi) \forall i,|\Theta\rangle$, as well as its degenerate partner state

$$
|-\Theta\rangle=P_{z}|\Theta\rangle=\left|-\theta_{1}, \ldots-\theta_{n}\right\rangle,
$$

will be ground states of $H$ when Eqs. (3) and (4) are fulfilled. ${ }^{20}$ Of course, they can be GS also in other cases ${ }^{10,11}$ by suitably adjusting the relative signs of the $\theta_{i}$ (see Sec. III A). Equation (4) are in fact the stationary conditions for the energy (5) at fixed $b^{i}, v_{\mu}^{i j}$, representing the mean field equations which ensure stability of $| \pm \Theta\rangle$ against one-spin excitations.

Equation (3) warrant that $| \pm \Theta\rangle$ will be exact eigenstates by canceling the residual matrix elements linking $| \pm \Theta\rangle$ with two-spin excitations and have a clear meaning within the random phase approximation ${ }^{24,25}$ (RPA): If satisfied, $\forall i, j$ the RPA vacuum will coincide with the mean-field state. More explicitly, the zero temperature RPA matrix (whose eigenvalues are the RPA energies) is

$$
\begin{gathered}
\mathcal{H}_{\mathrm{RPA}}=\left(\begin{array}{cc}
A & B^{-} \\
-B^{-} & -A
\end{array}\right), \quad A_{i j}=\lambda_{i} \delta_{i j}+B_{i j}^{+}, \\
B_{i j}^{ \pm}=-\frac{1}{2} \sqrt{s_{i} s_{j}}\left(v_{x}^{i j} \cos \theta_{i} \cos \theta_{j}+v_{z}^{i j} \sin \theta_{i} \sin \theta_{j} \pm v_{y}^{i j}\right),
\end{gathered}
$$

where $m \lambda_{i}$ are the eigenvalues of the local mean field Hamiltonian $b^{i} s_{i}^{z}-\Sigma_{j, \mu} v_{\mu}^{i j}\left\langle s_{j}^{\mu}\right\rangle_{\Theta} s_{i}^{\mu}=\lambda_{i} z_{i}^{z^{\prime}}$ and $B_{i j}^{ \pm}$the elements associated with the dispersion $\left(s_{i}^{+} s_{j}^{-}\right)$and creation $\left(s_{i}^{+} s_{j}^{+}\right)$of spin excitations respectively. Equation (3) is then equivalent to the condition $B^{-}=0$, implying no RPA corrections to the mean-field vacuum.

From Eq. (3) it is seen that a uniform eigenstate with $\theta_{i}$ $=\theta \forall i$ becomes feasible if the anisotropy ratio

$$
\chi \equiv \frac{v_{y}^{i j}-v_{z}^{i j}}{v_{x}^{i j}-v_{z}^{i j}}=\cos ^{2} \theta,
$$

is constant for all pairs and satisfies $\chi>0$ [if $\chi>1$ (complex $\theta$ ), a global rotation of $\pi / 2$ around the $z$ axis will lead to $\chi \rightarrow 1 / \chi$ and $\theta$ real]. Equation (4) leads then to

$$
b^{i}=\sqrt{\chi} \sum_{j \neq i}\left(v_{x}^{i j}-v_{z}^{i j}\right) s_{j},
$$

if $\chi \in[0,1)$ (the opposite sign for all $b^{i}$ is obviously also feasible) and to $b^{i}$ arbitrary if $\chi=1$ ( $X X Z$ or $X X$ case, ${ }^{26}$ where $|0\rangle$ is an exact eigenstate $\left.\forall b^{i}\right)$. Any spin array with couplings satisfying Eq. (6) will then exhibit a uniform separable degenerate eigenstate $|\Theta\rangle=|\theta, \ldots, \theta\rangle$ if the fields $b^{i}$ at sites $i$ are tuned at the values (7). It will be a GS when $\left|v_{y}^{i j}\right|<v_{x}^{i j} \forall i, j$.

\section{B. Entanglement at factorizing fields}

In a finite array the exact GS of $H$ will not be in general exactly degenerate in the vicinity of the factorizing point, and will have a definite $S_{z}$ parity. The correct side limits at the factorizing field are then provided by the normalized definite parity states

$$
\left|\Theta^{ \pm}\right\rangle=\frac{|\Theta\rangle \pm|-\Theta\rangle}{\sqrt{2(1 \pm\langle-\Theta \mid \Theta\rangle)}},
$$

where $\langle-\Theta \mid \Theta\rangle=\prod_{i=1}^{n} \cos ^{2 s_{i}} \theta_{i}$ is the overlap between the degenerate separable eigenstates. The states (8) satisfy $P_{z}\left|\Theta^{ \pm}\right\rangle= \pm\left|\Theta^{ \pm}\right\rangle$and are obviously also exact eigenstates when Eqs. (3) and (4) are fulfilled.

These states are entangled, with Schmidt $\operatorname{rank}^{4} 2$ for any bipartition $(A, \bar{A})$ of the whole system ${ }^{20}$ (here $A$ denotes a subset of spins and $\bar{A}$ the complementary subset). Moreover, the reduced state of any subsystem of two or more spins can be effectively considered as a two-qubit mixed state with respect to any bipartition. ${ }^{20}$ The entanglement between any two subsystems can then be measured through the concurrence, a measure of entanglement originally introduced for two-qubit systems ${ }^{27}$ (where it can be exactly computed, see Sec. III D), and later extended to mixed states of general bipartite systems through the convex roof extension of the generalized pure state expression. ${ }^{28,29}$ The concurrence between any two spins $i, j$ in the states $\left|\Theta^{ \pm}\right\rangle$can be shown to $\mathrm{be}^{20}$

$$
C_{i j}^{ \pm}=\frac{\sqrt{\left(1-\cos ^{4 s_{i}} \theta_{i}\right)\left(1-\cos ^{4 s_{j}} \theta_{j}\right)}\left\langle-\Theta_{\overline{i j}} \mid \Theta_{\overline{i j}}\right\rangle}{1 \pm\langle-\Theta \mid \Theta\rangle},
$$

where $\left\langle-\Theta_{\overline{i j}} \mid \Theta_{\overline{i j}}\right\rangle=\Pi_{k \neq i, j} \cos ^{4 k} \theta_{k}$ denotes the complementary overlap. It will be appreciable for sufficiently small angles $\theta_{k}$ if $\theta_{i}, \theta_{j} \neq 0$.

On the other hand, the entanglement between one spin and the rest of the chain in the states (8) can be measured through the entropy $S_{i}=-\operatorname{Tr}\left[\rho_{i} \log \rho_{i}\right]$, where $\rho_{i}$ $=\operatorname{Tr}_{i}^{-}\left|\Theta^{ \pm}\right\rangle\left\langle\Theta^{ \pm}\right|$is the reduced density matrix of the spin at site $i$, or alternatively, through the pure state concurrence ${ }^{28}$ $C_{i}=\sqrt{2\left(1-\operatorname{Tr} \rho_{i}^{2}\right)}$. The latter provides an upper bound to the sum of all pairwise concurrences $C_{i j}$ stemming from site $i:^{20,30,31} C_{i}^{2} \geq \sum_{j \neq i} C_{i j}^{2}$. It fully determines $S_{i}$ when $s_{i}=1 / 2$ (Sec. III D). Its expression in the states (8) reads ${ }^{20}$ 


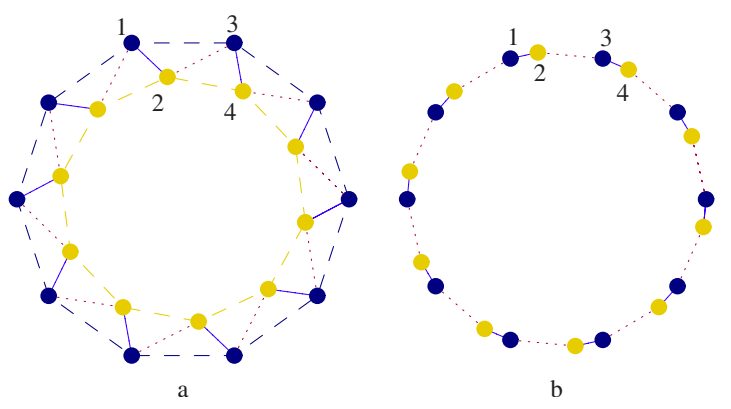

FIG. 1. (Color online) Schematic plot of (a) a system described by the couplings (11), representing two interacting cyclic chains and (b) the dimer chain of Eq. (12), a particular case of (a). Numbers indicate the notation used in Figs. 2 and 3.

$$
C_{i}^{ \pm}=\frac{\sqrt{\left(1-\cos ^{4 s_{i}} \theta_{i}\right)\left(1-\prod_{k \neq i} \cos ^{4 s_{k}} \theta_{k}\right)}}{1 \pm\langle-\Theta \mid \Theta\rangle},
$$

with $C_{i}^{ \pm} \approx \sqrt{1-\cos ^{4 s_{i}} \theta_{i}}$ if the overlap is neglected. The entanglement between $L$ and $n-L$ spins, as well as between any two sets of spins in the states (8) can also be exactly calculated. ${ }^{20}$

When $| \pm \Theta\rangle$ are GS, Eqs. (9) and (10) represent the actual side limits of the GS concurrences $C_{i j}$ and $C_{i}$ at the factorizing point, where a transition $\left|\Theta^{+}\right\rangle \rightarrow\left|\Theta^{-}\right\rangle$will take place as the field increases. ${ }^{16,20}$ The entanglement between two spins will then reach full range in its vicinity, provided $\left\langle-\Theta_{\overline{i j}} \mid \Theta_{\overline{i j}}\right\rangle \neq 0$ and $\theta_{i} \neq 0, \forall i, j[$ Eq. (9)].

When $\theta_{i}=\pi / 2 \forall i$ [corresponding for $v_{z}=0$ to the Ising case $v_{y}=0$ according to Eq. (6) $],\left| \pm \theta_{i}\right\rangle$ are orthogonal and $C_{i}^{ \pm}=1$ while $C_{i j}^{ \pm}=0 \forall i, j$. The previous effect becomes significant in the opposite limit of small $\theta_{i}$ (systems close to the $X X Z$ limit). We also remark that the uniform mixture of both definite parity states, $\rho_{0}=\frac{1}{2}\left(\left|\Theta^{+}\right\rangle\left\langle\Theta^{+}|+| \Theta^{-}\right\rangle\left\langle\Theta^{-}\right|\right)$, is also entangled and leads to attenuated concurrences ${ }^{20} C_{i j}^{0}$ $=C_{i j}^{-} \frac{\langle-\Theta \mid \Theta\rangle}{1+\langle-\Theta \mid \Theta\rangle}$ and $C_{i}^{0}=C_{i}^{-} \frac{\langle-\Theta \mid \Theta\rangle}{1+\langle-\Theta \mid \Theta\rangle}$.

\section{APPLICATION TO DIMER-TYPE CHAINS}

Let us now consider a pair of uniform interacting chains of the same size $m$ and spins $s_{\sigma}$, not necessarily equal. We can embed this system in a single nonuniform chain of even size $n=2 m$ assigning odd (even) sites to the first (second) chain, as schematically depicted in Fig. 1 (left), such that $\sigma=o, e$. We may then consider

$$
v_{\mu}^{i j}=v_{\mu}^{\sigma_{i}}(j-i)
$$

where $\sigma_{i}=o, e$ indicates the parity of the site, such that $v_{\mu}^{\sigma}(l)$ represents the interchain (internal) couplings for $l$ odd (even). Accordingly, $v^{o}(l)=v^{e}(-l)$ for $l$ odd and $v^{\sigma}(l)=v^{\sigma}(-l)$ for $l$ even [but $v^{o}(l) \neq v^{e}(l)$ in general]. In the cyclic case $v^{\sigma}(-l)=v^{\sigma}(n-l) \forall \sigma, l$.

An important example of this type is that of a dimer chain with just nearest neighbor couplings (Fig. 1, right), where $v_{\mu}^{\sigma}(l)=v_{\mu}^{\sigma} \delta_{l, \pm 1}$ :

$$
H_{d}=\sum_{i=1}^{n / 2}\left[b^{2 i-1} s_{2 i-1}^{z}+b^{2 i} s_{2 i}^{z}-\sum_{\mu=x, y, z} v_{\mu}^{o} s_{2 i-1}^{\mu} s_{2 i}^{\mu}+v_{\mu}^{e} s_{2 i}^{\mu} s_{2 i+1}^{\mu}\right] .
$$

Here $v_{\mu}^{e}$ can be considered as the (weak) couplings between dimers and $v_{\mu}^{o}$ the (strong) internal couplings, the system becoming dimerized (i.e., an array of independent spin pairs) for $v_{\mu}^{e} \rightarrow 0$ (see also Sec. III C).

A different example of Eq. (11), which nonetheless will exhibit factorization properties similar to those of Eq. (12) (see below), is a pair of arrays with no internal couplings interacting through a constant full range coupling: $v_{\mu}^{\sigma}(l)$ $=2 v_{\mu} / n \forall l$ odd and 0 otherwise such that

$$
H_{p}=b^{o} S_{o}^{z}+b^{e} S_{e}^{z}-\frac{1}{n} \sum_{\mu=x, y, z} v_{\mu} S_{o}^{\mu} S_{e}^{\mu},
$$

where $S_{o, e}^{\mu}=\Sigma_{l \text { odd }} s_{l}^{\mu}$ are the total spin components of each array and we have assumed a constant field is applied to each of them. This system is obviously equivalent to an interacting pair of spins $S_{o}=\frac{1}{2} n s_{o}$ and $S_{e}=\frac{1}{2} n s_{e}$ if restricted to the maximum spin multiplet. As in the Lipkin model, ${ }^{32}$ the $1 / n$ scaling ensures here a bounded intensive energy $\langle H\rangle / n$ for $n \rightarrow \infty$ and fixed $v_{\mu}$.

\section{A. Uniform separable eigenstates}

In the general case (11) with cyclic boundary conditions, a separable eigenstate with a common angle $\theta_{i}=\theta \forall i$ is then feasible if Eq. (6) holds for any connected pair, i.e., $\chi$ $=\frac{v_{y}^{\sigma}(l)-v_{z}^{\sigma}(l)}{v_{x}^{\sigma}(l)-v_{z}^{\sigma}(l)} \in[0,1)$ and constant $\forall l$, and there is a uniform field $b^{i}=b^{\sigma_{i}}$ in each subchain given by

$$
b^{\sigma}=\sqrt{\chi} \sum_{\sigma^{\prime}=o, e} v^{\sigma \sigma^{\prime}} s_{\sigma^{\prime}}, \quad \sigma=o, e,
$$

where $v^{\sigma \sigma}=\Sigma_{l \text { even }} v_{x}^{\sigma}(l)-v_{z}^{\sigma}(l)$ and $v^{o e}=\Sigma_{l \text { odd }} v_{x}^{\sigma}(l)-v_{z}^{\sigma}(l)$ $=v^{e o}$, with $b^{o}=b^{e}$ if $s_{o}=s_{e}$. Such uniform eigenstate is also feasible for a similar open chain provided a nonuniform field, as determined by Eq. (7), is applied. For short range couplings this will imply just boundary corrections. The ensuing states $| \pm \Theta\rangle$ will be GS if $\left|v_{y}^{\sigma}(l)\right|<v_{x}^{\sigma}(l) \forall \sigma, l$.

The definite parity states (8) will then lead to a finite concurrence (9) for any spin pair, which will depend on the parity of the sites but not on their separation: The odd-odd $\left(C_{o o}^{ \pm}\right)$, even-even $\left(C_{e e}^{ \pm}\right)$, and odd-even $\left(C_{o e}^{ \pm}\right)$concurrences will be given, according to Eq. (9), by

$$
C_{\sigma \sigma}^{ \pm}=\frac{\left(1-\chi^{\left.2 s_{\sigma}\right)} \chi^{S-2 s_{\sigma}}\right.}{1 \pm \chi^{S}}, \quad C_{o e}^{ \pm}=\sqrt{C_{o o}^{ \pm} C_{e e}^{ \pm}},
$$

where $S=\frac{1}{2} n\left(s_{o}+s_{e}\right)$ is the total spin. The range of the entanglement between two spins will then increase as the factorizing fields (14) are approached in each subchain, becoming independent of the coupling range and separation. If $s_{o}$ $=s_{e}$, obviously $C_{o o}=C_{e e}=C_{o e}$.

$C_{\sigma \sigma}^{ \pm}$will be appreciable for sufficiently small $X Y$ anisotropies: If $\chi \approx 1-\delta / S$ then $\chi^{S} \approx e^{-\delta}$ for small $\delta / S$. In fact, for $\delta \rightarrow 0$ ( $X X$ limit) $C_{\sigma \sigma}^{+} \rightarrow 0$ but $C_{\sigma \sigma}^{-} \rightarrow 2 s_{\sigma} / S$ (i.e., $2 / n$ for $s_{o}$ 
$=s_{e}$, which is the maximum attainable value when all pairs are equally entangled), ${ }^{33}$ as $\left|\Theta^{+}\right\rangle \rightarrow|0\rangle$ but $\left|\Theta^{-}\right\rangle$approaches the entangled $W$-type state ${ }^{34} \propto \Sigma_{i} s_{i}^{+}|0\rangle$. In the opposite limit (Ising case $\chi=0),| \pm \Theta\rangle$ become orthogonal and $C_{\sigma \sigma}=0$.

In the dimer chain (12), the uniform separable eigenstate becomes then feasible if there is a common anisotropy $\chi$ $=\frac{v_{y}^{\sigma}-v_{z}^{\sigma}}{v_{x}^{\sigma}-v_{z}^{\sigma}} \in[0,1)$ for $\sigma=o, e$ and the fields are chosen as [Eq. (14)]

$$
b^{o}=\sqrt{\chi} v^{o e} s_{e}, \quad b^{e}=b^{o} s_{o} / s_{e},
$$

where $v^{o e}=v_{x}^{o}+v_{x}^{e}-v_{z}^{o}-v_{z}^{e}$. In an open chain we should just add the border corrections $b^{1}=\frac{1}{2} b^{o}, b^{n}=\frac{1}{2} b^{e}$ according to Eq. (7). Thus, in the ferromagnetic-type case $\left|v_{y}^{\sigma}\right| \leq v_{x}^{\sigma}$ for $\sigma$ $=o, e$, its GS will become uniform at the factorizing fields (16), regardless of the ratio $v_{x}^{e} / v_{x}^{o}$ (as long as it is nonzero), loosing there all signatures of a dimerized structure and leading to the full range concurrences (15) as side limits.

Let us also remark that for the nearest neighbor couplings of Eq. (12), the antiferromagnetic case $v_{x}^{\sigma}<0 \forall \sigma$ can be brought back to the previous case by means of local rotations of angle $\pi$ around the $z$ axis at even sites (implying $s_{i}^{\mu} \rightarrow$ $(-1)^{i+1} s_{i}^{\mu}$ and hence $v_{\mu}^{\sigma} \rightarrow-v_{\mu}^{\sigma} \forall \sigma$ for $\left.\mu=x, y\right)$. A uniform separable eigenstate $|\nearrow \nearrow \ldots\rangle \equiv \mid \theta \theta$... in the rotated system corresponds then to an alternating solution $\theta_{i}=(-1)^{i+1} \theta$ (Neél-type state $|\nearrow \nwarrow \nearrow \nwarrow \ldots\rangle \equiv|\theta,-\theta, \theta \ldots\rangle)$ in the original system. Note that this holds for arbitrary spins $s_{\sigma}$ (equal or distinct). Separability (but not entanglement) in the $s_{o}$ $=s_{e}=1 / 2$ dimer chain was discussed in Ref. 19, with the correct treatment for general antiferromagnetic couplings discussed in detail in Refs. 17 and 18.

For even $m=n / 2$ (to avoid frustration effects), ${ }^{18}$ the mixed case $v_{x}^{o}>0, v_{x}^{e}<0$ (or vice-versa) can also be recast as a ferromagnetic case $v_{x}^{\sigma}>0 \forall \sigma$ by means of local rotations of $\pi$ around the $z$ axis in even sites of both subchains $\left[s_{2 i+k}^{\mu}\right.$ $\rightarrow(-1)^{i+1} s_{2 i+k}^{\mu}$ for $k=-1,0$ and $\mu=x, y$, implying $\left.v_{\mu}^{e} \rightarrow-v_{\mu}^{e}\right]$. The uniform solution corresponds here to $\theta_{2 i+k}=(-1)^{i+1} \theta$ for $k=-1,0$ in the original mixed system, i.e., ${ }^{18,19}$ $|\nearrow \nearrow \nwarrow \nwarrow \ldots\rangle \equiv|\theta, \theta,-\theta,-\theta, \theta, \ldots\rangle$. Hence, for even $n / 2$ we may always assume $v_{x}^{\sigma}>0 \forall \sigma$ in Eq. (12).

In the system (13), the same uniform separable eigenstate becomes feasible if $\chi=\frac{v_{y}-v_{z}}{v_{x}-v_{z}} \in[0,1)$ and the fields are set at the values (16), with $v^{o e}=v_{x}-v_{z}$ [Eq. (14)]. We may again assume $v_{x} \geq 0$ since its sign can be changed replacing $S_{e}^{\mu} \rightarrow$ $-S_{e}^{\mu}$ for $\mu=x, y$. This system will exhibit just three different GS pairwise concurrences $\left(C_{o o}, C_{e e}, C_{o e}\right)$ for any $b^{o}$ and $b^{e}$, which will approach the same limits (15) at the fields (16).

\section{B. Alternating separable eigenstates}

In the case of two interacting subchains with no internal couplings, such as Eqs. (12) and (13), we may also consider the possibility of different and controllable uniform angles $\theta_{o}, \theta_{e}$ (with $\left|\theta_{o}\right| \neq\left|\theta_{e}\right|$ ) in each subchain, i.e.,

$$
|\Theta\rangle=\left|\theta_{o} \theta_{e} \theta_{o} \theta_{e} \ldots\right\rangle,
$$

through an alternating field $b^{o} \neq b^{e}$. For simplicity we will consider $X Y$ couplings $\left(v_{z}^{i j}=0\right)$. According to Eqs. (3) and (4), such a solution is feasible if for $\sigma=o, e$ and $l$ odd,

$$
\chi=\frac{v_{y}^{\sigma}(l)}{v_{x}^{\sigma}(l)}=\cos \theta_{o} \cos \theta_{e}, \quad b^{\sigma}=v^{o e} \frac{\sin \theta_{\sigma}}{\tan \theta_{\bar{\sigma}}} s_{\bar{\sigma}},
$$

where $v^{o e}=\Sigma_{l \text { odd }} v_{x}^{\sigma}(l)$ and $\bar{\sigma} \neq \sigma$ [i.e., $v^{o e}=v_{x}^{o}+v_{x}^{e}$ in Eq. (12) and $v^{o e}=v_{x}$ in Eq. (13)]. This implies

$$
b^{o} b^{e}=\chi\left(v^{o e}\right)^{2} s_{o} s_{e}
$$

$$
\cos ^{2} \theta_{\sigma}=\frac{\chi^{2}+\widetilde{b}_{\sigma}^{2}}{1+\widetilde{b}_{\sigma}^{2}}, \quad \tilde{b}_{\sigma} \equiv \frac{b^{\sigma}}{v^{o e} s_{\bar{\sigma}}} .
$$

Hence, for fields $b^{o}, b^{e}$ satisfying Eq. (18) we obtain a separable eigenstate with alternating angles $\theta_{o}, \theta_{e}$ determined by Eq. (19). Since one of the fields is now free, we may adjust in such system the individual angles and thus the internal $\left(C_{o o}^{ \pm}, C_{e e}^{ \pm}\right)$and interchain $\left(C_{o e}^{ \pm}=C_{e o}^{ \pm}\right)$pairwise concurrences at separability, given now by

$$
C_{\sigma \sigma}^{ \pm}=\frac{\left(1-\chi_{\sigma}^{2 s_{\sigma}}\right) \chi_{\sigma}^{S_{\sigma}-2 s_{\sigma}} \chi_{\bar{\sigma}}^{S_{\bar{\sigma}}}}{1 \pm \chi_{\sigma}^{S_{\sigma}} \chi_{\bar{\sigma}}^{S_{\bar{\sigma}}}}, \quad C_{o e}^{ \pm}=\sqrt{C_{o o}^{ \pm} C_{e e}^{ \pm}},
$$

where $\chi_{\sigma} \equiv \cos ^{2} \theta_{\sigma}$ and $S_{\sigma}=n s_{\sigma} / 2$. If $\left|b^{o}\right|>\left|b^{e}\right|$ and $s_{o}=s_{e}$, $C_{o o}^{ \pm}<C_{o e}^{ \pm}<C_{e e}^{ \pm}$, despite the absence of even-even direct coupling $\left(v^{e e}=v^{o o}=0\right)$. For $\tilde{b}^{o}=\tilde{b}^{e}$ we recover Eqs. (15) and (16). The values of $C_{\sigma \sigma^{\prime}}^{ \pm}$depend now on the ratio $\eta=\tilde{b}^{o} / \tilde{b}^{e}\left[\tilde{b}_{o}\right.$ $=\sqrt{\eta \chi}, \tilde{b}_{e}=\sqrt{\chi / \eta}$ when Eq. (18) holds]. For $\eta \gg 1$, $\cos \theta_{o}$ $\rightarrow 1$ but $\cos \theta_{e} \rightarrow \chi$, implying that in this limit $C_{e e}^{ \pm}$remains finite at the factorizing field, while $C_{o o}^{ \pm}$and $C_{o e}^{ \pm}$vanish. Note also that $\theta_{\sigma}$ is a decreasing function of $\tilde{b}_{\sigma}$.

In the ferromagnetic case $v_{x}^{\sigma}>0, \theta_{o}$ and $\theta_{e}$ have both the same sign. For antiferromagnetic couplings $v_{x}^{\sigma}<0 \forall \sigma$ in the dimer chain (12), we would have instead $\theta_{o}>0$ and $\theta_{e}<0$ (or vice-versa), whereas in the mixed case $v_{x}^{e} v_{x}^{o}<0,|\Theta\rangle$ $=\left|\theta_{o} \theta_{e},-\theta_{o},-\theta_{e}, \theta_{o} \theta_{e}, \ldots\right\rangle$, as previously discussed. Border corrections $b^{1}=\frac{1}{2} b^{o}, b^{n}=\frac{1}{2} b^{e}$ would also apply in an open dimer chain.

The concurrence between one spin and the rest of the chain $C_{\sigma_{i}} \equiv C_{i}$, will be given at separability by [Eq. (10)]

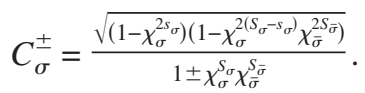

\section{Spin 1/2 pair}

We may explicitly verify the previous expressions (valid for general $n)$ in the "two-qubit" case $\left(s_{o}=s_{e}=1 / 2, n=2\right)$, which also represents the $v_{\mu}^{e} \rightarrow 0$ limit in the spin $1 / 2$ dimer chain (12). Setting $v_{ \pm}=\frac{1}{4}\left(v_{x} \pm v_{y}\right) \geq 0$ and $b_{ \pm}=\frac{1}{2}\left(b^{o} \pm b^{e}\right)$, with $b^{o}=b^{1}, b^{e}=b^{2}$, the eigenstates and energy levels of Hamiltonian (1) become in this case

$$
\left|\Psi_{ \pm}^{-}\right\rangle=\alpha_{\mp}^{-}|\uparrow \downarrow\rangle \pm \alpha_{ \pm}^{-}|\downarrow \uparrow\rangle, \quad E_{ \pm}^{-}=\frac{1}{4} v_{z} \mp \sqrt{b_{-}^{2}+v_{+}^{2}},
$$




$$
\left|\Psi_{ \pm}^{+}\right\rangle=\alpha_{\mp}^{+}|\uparrow \uparrow\rangle \pm \alpha_{ \pm}^{+}|\downarrow \downarrow\rangle, \quad E_{ \pm}^{+}=-\frac{1}{4} v_{z} \mp \sqrt{b_{+}^{2}+v_{-}^{2}},
$$

where $\left(\alpha_{ \pm}^{\nu}\right)^{2}=\frac{1}{2}\left(1 \pm \frac{b_{\nu}}{\sqrt{b_{\nu}^{2}+v_{-\nu}^{2}}}\right)$ and the superscript $\nu= \pm$ indicates the $S_{z}$ parity. The GS corresponds to $\left|\Psi_{+}^{-}\right\rangle$or $\left|\Psi_{+}^{+}\right\rangle$, with $E_{+}^{-}$and $E_{+}^{+}$crossing precisely when the factorizing conditions (3) and (4) hold. At this point $\left|\Psi_{+}^{ \pm}\right\rangle$become the states (2). In particular, for an homogeneous field $\left(b_{-}=0, b_{+}=b\right), E_{+}^{-}$ $=E_{+}^{+}$when $b=\frac{1}{2} \sqrt{\chi}\left(v_{x}-v_{z}\right)\left[\right.$ Eq. (7)], whereas for $v_{z}=0$ they cross when $b^{o} b^{e}=\frac{1}{4} \chi v_{x}^{2}$ [Eq. (18)]. It is then explicitly verified that the states (2) are the true side limits at the crossing point, with separability arising just from the crossing of these two states. Factorization corresponds then to the quantum critical point of the spin $1 / 2$ pair. It should be also noticed that $\left|\Psi_{+}^{ \pm}\right\rangle$can here be always written as projected states (2) using suitable angles $\left(\tan ^{2} \frac{1}{2} \theta_{1}=\frac{\alpha_{-}^{+} \alpha_{-}^{-}}{\alpha_{+}^{+} \alpha_{+}^{-}}, \tan ^{2} \frac{1}{2} \theta_{2}=\frac{\alpha_{-}^{+} \alpha_{+}^{-}}{\alpha_{+}^{+} \alpha_{-}^{-}}\right)$. The concurrence $C_{12}=\sqrt{2\left(1-\operatorname{Tr} \rho_{1}^{2}\right)}$ in the states $\left|\Psi_{\nu}^{ \pm}\right\rangle$reads

$$
C_{12}^{ \pm}=2\left|\alpha_{+}^{ \pm} \alpha_{-}^{ \pm}\right|=\left|v_{\mp}\right| / \sqrt{b_{ \pm}^{2}+v_{\mp}^{2}},
$$

and coincides with both general results (9) and (10) for the present case $\left[C_{12}^{ \pm}=\left|\sin \theta_{1} \sin \theta_{2}\right| /\left(1 \pm \cos \theta_{1} \cos \theta_{2}\right)\right]$.

In the spin $1 / 2$ dimer chain (12), Eq. (24) represents the limit of the concurrence $C_{2 i-1,2 i}$ for $v_{\mu}^{e} \rightarrow 0$. This implies that its GS will become fully dimerized (i.e., an array of maximally entangled pairs) at zero field, since in this case $C_{12}^{ \pm}$ $=1$ and all eigenstates $\left|\Psi_{\nu}^{ \pm}\right\rangle$are Bell states. However, at finite fields, $C_{12}^{ \pm}=1$ only if $b_{ \pm}=0\left(b^{e}=\mp b^{\circ}\right)$, in which case just half of the eigenstates remain maximally entangled. For $b^{o, e}>0$, maximum entanglement $\left(C_{12}=1\right)$ for $v_{\mu}^{e} \rightarrow 0$ will then arise just for an homogeneous field $b_{+}=b$ lower than the factorizing field, i.e., when the pair GS is antiparallel $\left(\left|\Psi_{+}^{-}\right\rangle\right)$.

Let us finally notice that for $\nu= \pm$,

$$
\left\langle s_{i}^{z}\right\rangle_{\nu} \equiv\left\langle\Psi_{+}^{\nu}\left|s_{i}^{z}\right| \Psi_{+}^{\nu}\right\rangle=-\frac{1}{2}(\nu)^{i+1} b_{\nu} / \sqrt{b_{\nu}^{2}+v_{-\nu}^{2}},
$$

implying $\left\langle s_{1}^{z}\right\rangle_{+}=\left\langle s_{2}^{z}\right\rangle_{+}$but $\left\langle s_{1}^{z}\right\rangle_{-}=-\left\langle s_{2}^{z}\right\rangle_{-}$, i.e., opposite magnetizations for negative $S_{z}$ parity (see below).

\section{Results}

Figures 2 and 3 depict illustrative results for the GS pairwise concurrence $C_{i j}$ in a finite spin 1/2 dimer chain described by Eq. (12) with cyclic $X Y$ couplings $\left(v_{z}^{\sigma}=0\right)$. We have set $v_{\mu}^{e}=\alpha v_{\mu}^{o}$, with $v_{y}^{\sigma}=\chi v_{x}^{\sigma}$ for $\sigma=o, e$. Full exact results for finite $n$ can in this case be obtained through the JordanWigner transformation (see Appendix). In this system $C_{i j}$ $=C_{1, j-i+1}\left(C_{2, j-i+2}\right)$ for $i$ odd (even).

The reduced density matrix for a pair of spins $i, j$ will commute with the pair parity $e^{i \pi\left(s_{i}^{z}+s_{j}^{z}-1\right)}$, being then of the form $(\langle\ldots\rangle$ denotes here the GS average)

$$
\rho_{i j}=\frac{1}{4}+\left\langle s_{i}^{z}\right\rangle s_{i}^{z}+\left\langle s_{j}^{z}\right\rangle s_{j}^{z}+4 \sum_{\mu=x, y, z}\left\langle s_{i}^{\mu} s_{j}^{\mu}\right\rangle s_{i}^{\mu} s_{j}^{\mu} .
$$

The GS pairwise concurrence $C_{i j}$ can then be evaluated as ${ }^{27}$ $2 \lambda_{\max }-\operatorname{Tr} R$, with $\lambda_{\max }$ the greatest eigenvalue of the matrix $R=4 \sqrt{s_{i}^{y} s_{j}^{y} \rho_{i j} s_{i}^{y} s_{j}^{y} \rho_{i j}}$, and reads

$$
C_{i j}=\operatorname{Max}\left[C_{i j}^{+}, C_{i j}^{-}, 0\right],
$$

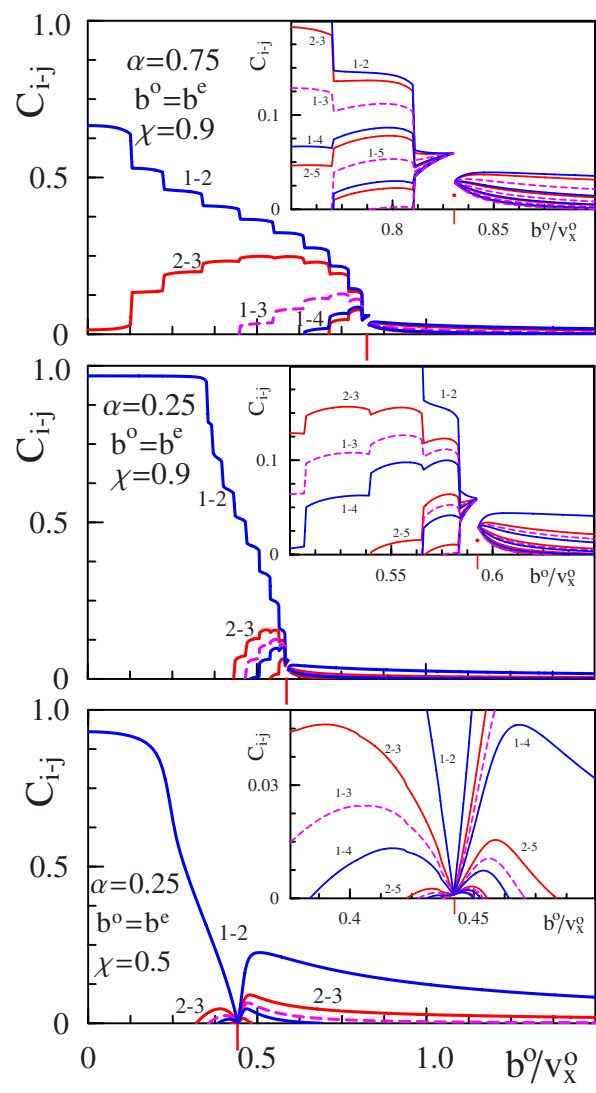

FIG. 2. (Color online) Concurrences between spins $i, j$ vs magnetic field in a spin $1 / 2 X Y$ dimer chain (Eq. (12)) for two values of $\alpha \equiv v_{\mu}^{e} / v_{\mu}^{o}$ and of the anisotropy $\chi \equiv v_{y}^{\sigma} / v_{x}^{\sigma}$. The field is here uniform, with $n=20$ spins. All $C_{i j}$ approach the same side limits (15) (which are independent of $\alpha$ ) at the factorizing field (28) (vertical bar), as seen in the insets (blow up of main plot), changing from antiparallel to parallel and exhibiting there the same finite step. The red dot at $b=b_{s}$ indicates the concurrence $C_{i j}^{0}$ in the mixture of both definite parity ground states.

$$
C_{i j}^{ \pm}=2\left[\left|\left\langle s_{i}^{x} s_{j}^{x} \mp s_{i}^{y} s_{j}^{y}\right\rangle\right|-\sqrt{\left(\frac{1}{4} \mp\left\langle s_{i}^{z} s_{j}^{z}\right\rangle\right)^{2}-\frac{1}{4}\left\langle s_{i}^{z} \mp s_{j}^{z}\right\rangle^{2}}\right],
$$

being parallel ${ }^{14}$ (i.e., as in the states $\left.|\uparrow \uparrow\rangle+|\downarrow \downarrow\rangle\right)$ if $C_{i j}^{+}>0$ and antiparallel $(|\uparrow \downarrow\rangle-|\downarrow \uparrow\rangle)$ if $C_{i j}^{-}>0$ (just one of $C_{i j}^{ \pm}$can be positive). The entanglement of formation of the pair can then be obtained as $^{27} \quad S_{i j}=-\Sigma_{\nu= \pm} p_{\nu} \log _{2} p_{\nu}$, where $p_{ \pm}$ $=\frac{1}{2}\left(1 \pm \sqrt{1-C_{i j}^{2}}\right) . C_{i j}=S_{i j}=0$ (1) for a separable (maximally entangled) pair.

The case of a uniform field $b^{o}=b^{e}=b$ is depicted in Fig. 2 . Here $C_{1 j}=C_{2, j+1}$ for $j$ odd. At $b=0$ and for $\alpha=1$ (uniform chain), there is entanglement just between first neighbors $\left(C_{12}=C_{23}>0\right)$. For a small anisotropy $\chi=0.9$, as soon as the ratio $\alpha$ decreases below 1 the concurrence between weakly coupled pairs $\left(C_{23}\right)$ rapidly decreases (top panel), vanishing here already for $\alpha \lesssim 0.74$, whereas $C_{12}$ rapidly increases, practically reaching saturation for $\alpha=0.25$ (center panel). Hence, at zero field approximate dimerization is achieved already for low $\alpha$, the system becoming essentially an array of maximally entangled pairs in the antiparallel states $\left|\Psi_{+}^{-}\right\rangle$. 


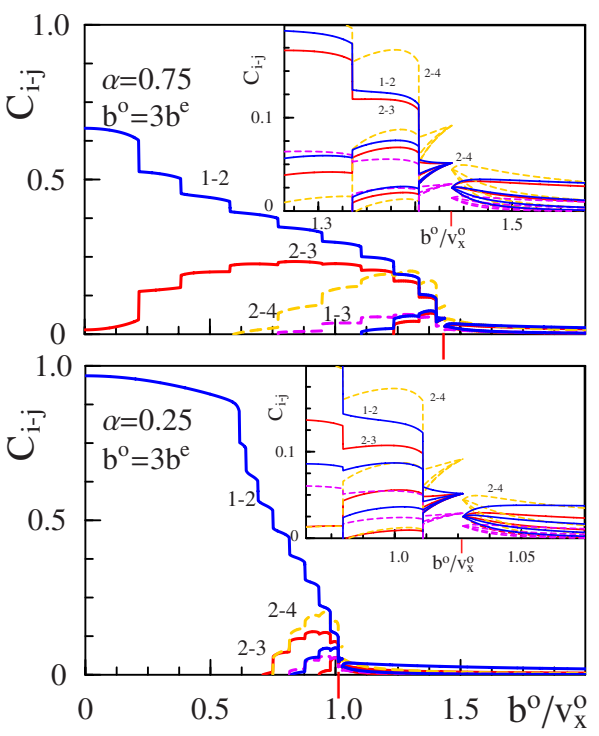

FIG. 3. (Color online) Same details as the top and center panel of Fig. 2 for the case of different fields at even and odd sites, with a fixed ratio $b^{o} / b^{e}=3$. Now odd-even, odd-odd, and even-even concurrences approach different common side limits at the factorizing field (29), with $C_{24}$ becoming the greatest concurrence in its vicinity.

The previous picture remains valid for weak finite fields. As seen in the top and central panels, increasing the uniform field destroys dimerization in a stepwise manner, the GS remaining almost unchanged until the first step, occurring at $b^{*} \approx \frac{1}{2} \sqrt{\chi} v_{x}^{o}(1-\alpha)$ for $\alpha$ not close to 1 . These steps, clearly visible in small chains with low anisotropy, reflect the $n / 2$ GS $S_{z}$-parity transitions (crossings between the lowest levels of opposite parity, ${ }^{16,19}$ which are close but not degenerate) taking place as the field increases when $\chi \in(0,1]$, as in the homogeneous $X Y$ chain. At the same time, the concurrence range increases as the last step is approached. The latter occurs precisely at the uniform factorizing field [Eq. (7)]

$$
b_{s}=\frac{1}{2} \sqrt{\chi} v_{x}^{o}(1+\alpha),
$$

where the dimer structure is completely lost and entanglement reaches full range: all pairs become equally entangled irrespective of separation or location, with $C_{i j}$ reaching the side limits (15) $\forall i \neq j\left(\lim _{b \rightarrow b_{s}^{ \pm}} C_{i j}=C_{i j}^{ \pm}\right)$, which are independent of $\alpha$ and hence the same in top and central panels. At this field all $C_{i j}$ exhibit the same finite discontinuity, changing from antiparallel $\left(b<b_{s}\right)$ to parallel $\left(b>b_{s}\right)$. For $\alpha \rightarrow 0$, $b^{*}$ and $b_{s}$ (first and last steps) merge at the two-qubit factorizing field $\frac{1}{2} \sqrt{\chi} v_{x}^{o}$.

For stronger fields $b>b_{s}$ we obtain a weak parallel concurrence, which for first and second neighbors persists for arbitrarily strong fields and can be described perturbatively. First (second) neighbors concurrences are first (second) order in $v_{x}^{\sigma} / b$ and given, up to $O\left(v_{x}^{\sigma} / b\right)^{2}$, by

$$
C_{12} \approx\left|\frac{v_{-}^{o}}{b}\right|-\frac{1}{2}\left(\alpha \frac{v_{-}^{o}}{b}\right)^{2}, \quad C_{23} \approx\left|\frac{\alpha v_{-}^{o}}{b}\right|-\frac{1}{2}\left(\frac{v_{-}^{o}}{b}\right)^{2},
$$

$$
C_{i, i+2} \approx\left|\frac{\alpha v_{-}^{o} v_{+}^{o}}{b b^{\sigma_{i}}}\right|-\frac{1}{2}\left(\frac{v_{-}^{o}}{b}\right)^{2}\left(1+\alpha^{2}\right)
$$

where $v_{ \pm}^{o}=\frac{1}{4}\left(v_{x}^{o} \pm v_{y}^{o}\right)=\frac{1}{4} v_{x}^{o}(1 \pm \chi)$ and $b=\frac{1}{2}\left(b^{o}+b^{e}\right)$. Note that a threshold value of $\alpha$ is required for a positive second neighbor concurrence for strong fields.

For higher anisotropies (lower $\chi$ ), the behavior becomes similar to that of larger systems. The GS parity transitions become less noticeable, as seen for $\chi=0.5$ in the bottom panel, and the pairwise concurrence side limits at the factorizing field are smaller. Nonetheless, the increase in the concurrence range in its vicinity remains clearly appreciable. Let us remark that for small separations $|i-j|$, the results for $C_{i j}^{ \pm}$ for $n=20$ at $\chi=0.5$ are already very close to those for $n$ $\rightarrow \infty$. We should also mention that as $\chi$ decreases, lower ratios $\alpha$ are required to achieve approximate dimerization at low fields (at $\chi=0.5$ and $b=0, C_{23}$ vanishes only for $\alpha$ $\lesssim 0.58$ ).

Figure 3 depicts the typical behavior for small anisotropies when different fields are applied at even at odd sites, with a fixed ratio $\eta=b^{o} / b^{e}=3$. The factorizing value for $b^{o}$ [Eq. (18)] is here

$$
b_{s}^{o}=\sqrt{\eta} b_{s}=\frac{1}{2} \sqrt{\eta \chi} v_{x}^{o}(1+\alpha) .
$$

At $b_{s}^{o}$ there are now three different limits for the concurrences at each side, $C_{o o}^{ \pm}, C_{e e}^{ \pm}$, and $C_{o e}^{ \pm}$, which represent the common side limits of $C_{1,2 j+1}, C_{2,2 j+2}$, and $C_{1,2 j}, C_{2,2 j+1} \forall j$ and are given by Eq. (20). They satisfy here

$$
C_{e e}^{ \pm} / C_{o o}^{ \pm}=(\chi+\eta) /\left(\chi+\eta^{-1}\right)>1,
$$

for $\eta>1$, implying $C_{e e}^{ \pm}>C_{o e}^{ \pm}$. In particular, $C_{24}$ [which approaches $C_{e e}^{ \pm}$for $\left.b^{o} \rightarrow\left(b_{s}^{o}\right)^{ \pm}\right]$clearly exceeds in the vicinity of $b_{s}^{o}$ both first neighbor concurrences $C_{12}$ and $C_{23}$, despite the absence of second-neighbor couplings.

It is also seen that $C_{12}$ is no longer nearly constant up to the first parity transition, which occurs now at $b^{o *}$ $\approx \frac{1}{2} \sqrt{\eta \chi} v_{x}^{o}(1-\alpha)$. This effect can be easily understood with the two-qubit concurrence (24): at low $\alpha, C_{12}$ is essentially described in the first region by the two-qubit expression (24) for $C_{12}^{-}$, which for $b_{-}=\frac{1}{2} b^{o}(1-1 / \eta) \neq 0$, is no longer constant and decreases as $b^{\circ}$ increases.

In order to highlight the universality of the limits at the factorizing field, we depict in Fig. 4 the pairwise concurrences in the system (13) for $s_{o}=s_{e}=1 / 2$, where the exact solution can be obtained through direct diagonalization in the $S_{o}=S_{e}=n / 2$ representation. In the $X Y$ case, $v^{o e}=v_{x}$ and the odd factorizing field at fixed ratio $b^{o} / b^{e}=\eta$ is $b_{s}^{o}=\frac{1}{2} v_{x} \sqrt{\eta \chi}$. There are now just three different concurrences at all fields, $C_{o o}=C_{1,2 j+1}, C_{e e}=C_{2,2 j+2}$, and $C_{o e}=C_{1,2 j}=C_{e o}$ ( $j$ independent), which approach the same limits of Figs. 2 and 3 [Eqs. (15) and (20)] at the factorizing field, since the latter depend solely on $\chi$ and the field ratio $\eta$. They are here comparable to the values away from the factorizing field, since the monogamy bound on $\Sigma_{j \neq i} C_{i j}^{2}$ entails $C_{\sigma \sigma^{\prime}}=O(1 / n)$ in this symmetric system. ${ }^{33}$ In the case considered $C_{o o}$ and $C_{e e}$ are in fact maximum at the factorizing field. Note again that for 


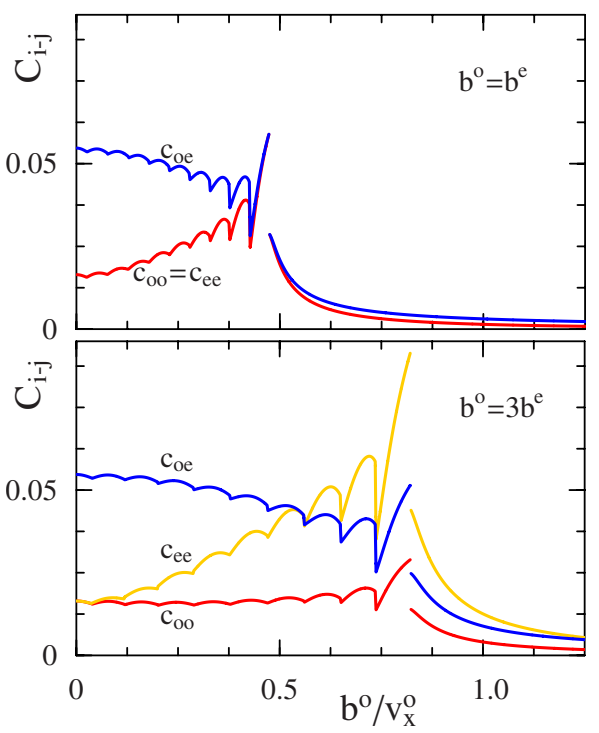

FIG. 4. (Color online) Concurrences between spins $i, j$ vs magnetic field in a system with constant full range couplings between even and odd sites, described by Hamiltonian (13). We have set again $\chi=0.9, n=20$, and a uniform (alternating) field in the top (bottom) panels. Odd-even, odd-odd, and even-even concurrences approach at the factorizing field exactly the same side limits as those of Figs. 2 and 3, respectively, which are here of the same order as the values outside this field.

$b^{o} / b^{e}>1, C_{e e}>C_{o e}$ in the vicinity of $b_{s}^{o}$, despite the absence of even-even couplings.

Finally, we depict in Fig. 5 the site magnetizations $M_{\sigma_{i}}$ $\equiv\left\langle s_{i}^{z}\right\rangle$ together with the concurrence $C_{\sigma_{i}} \equiv C_{i}$ between one spin and the rest of the chain [Eq. (10)]. For a spin $1 / 2$ chain with $\left[H, P_{z}\right]=0$, both quantities are strictly related, since the

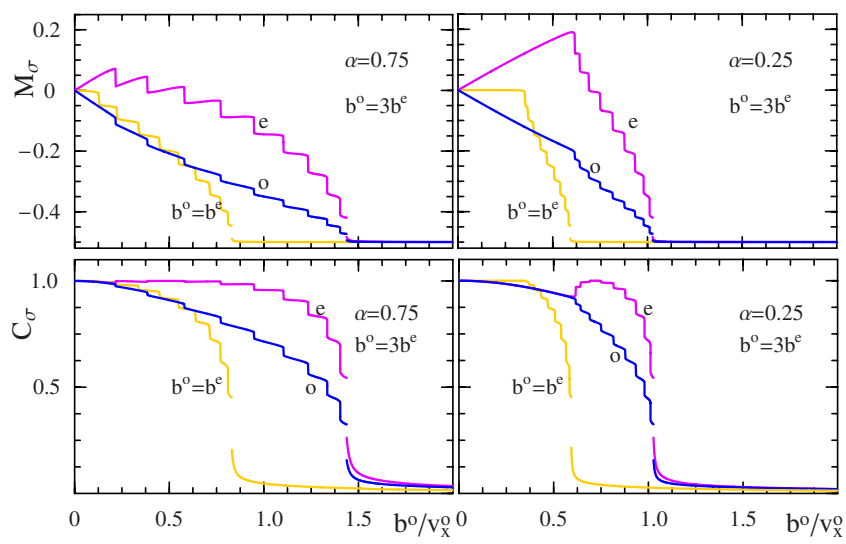

FIG. 5. (Color online) Magnetization at even and odd sites $M_{\sigma} \equiv\left\langle s_{\sigma}^{z}\right\rangle$ (top panels) and the concurrence $C_{\sigma} \equiv C_{i}$ [Eq. (30)] between the site and the rest of the chain (bottom panels), in the dimer chain of Figs. 2 and 3 with $\chi=0.9$. Results for an alternating field with fixed ratio $b^{o} / b^{e}=3$ and for a uniform field are depicted. The discontinuities at the factorizing field are explicitly shown. The "dimer phase" (fields below the first transition) presents opposite magnetizations for a nonuniform field and leads to a nonmonotonous behavior of $C_{e}$ after this transition. reduced density matrix for one spin in a state with definite parity is diagonal in the $s_{z}$ basis $\left(\rho_{i}=\frac{1}{2}+2\left\langle s_{i}^{z}\right\rangle s_{i}^{z}\right.$ as $\left\langle s_{i}^{\mu}\right\rangle=0$ for $\mu=x, y)$ and hence

$$
C_{i}=\sqrt{2\left(1-\operatorname{Tr} \rho_{i}^{2}\right)}=\sqrt{1-4\left\langle s_{i}^{z}\right\rangle^{2}} .
$$

Thus, $C_{i}=1$ when $\left\langle s_{i}^{z}\right\rangle=0$ (zero field). At the factorizing field it approaches the side limits (10). The ensuing entanglement entropy can be evaluated as $S_{i}=-\Sigma_{\nu= \pm} p_{\nu} \log _{2} p_{\nu}$, with $p_{ \pm}$ $=\frac{1}{2}\left(1 \pm \sqrt{1-C_{i}^{2}}\right)$.

While for a uniform field the even and odd site magnetizations coincide and decrease stepwise as the field increases, approaching $-1 / 2$ for strong fields, for nonuniform fields they first acquire opposite signs $\left(M_{o}=-M_{e}\right)$ in the "dimer phase," i.e., before the first parity transition. Here the magnetization is essentially described by the two-qubit result (25), which yields $M_{e}=-M_{o}>0$ in the state $\left|\Psi_{+}^{-}\right\rangle$if $b^{o}>b^{e}$. Accordingly, $M_{e}$ first increases as $b^{o}$ [and hence $b^{e}$ and $b_{-}$in (25)] increases, in close agreement with Eq. (25). After the first transition, $M_{e}$ starts to decrease, crossing 0 and approaching $-1 / 2$ (together with $M_{o}$ ) for strong fields, even though it may still increase between transitions.

This entails a nonmonotonous behavior of $C_{e}$ for increasing fields, particularly appreciable for low $\alpha$, where $C_{e}$ saturates again $\left(C_{e}=1\right)$ at a finite field, i.e., when $M_{e}$ vanishes. At the factorizing field $C_{\sigma}$ approaches the limits (21), which are independent of $\alpha$, with the magnetization step there given by $^{20} \Delta M_{i} \equiv\left\langle s_{i}^{z}\right\rangle_{-}-\left\langle s_{i}^{z}\right\rangle_{+}=\frac{\sin ^{2} \theta_{i}\left(-\Theta_{i} \Theta \Theta_{i}\right\rangle}{1-\langle-\Theta \mid \Theta\rangle^{2}}$. For strong fields the behavior of $M_{\sigma}$ and $C_{\sigma}$ can again be described perturbatively: we obtain $M_{o} \approx M_{e} \approx-\frac{1}{2}\left[1-\left(\frac{v_{-o}^{o}(1+\alpha)}{b}\right)^{2}\right]$ with $C_{o} \approx C_{e}$ $\approx\left|\frac{v_{-}^{o}(1+\alpha)}{b}\right|$.

\section{CONCLUSIONS}

We have first determined the factorization conditions for general dimer-type arrays with $X Y Z$ couplings in general transverse fields. We have also examined the entanglement properties of the associated definite parity states, which constitute the actual GS side limits at separability in a finite system, showing that weak but nonzero full range pairwise entanglement can be reached in the vicinity of factorizing fields. The possibility of an alternating and field dependent separable GS through a nonuniform field along a separability curve [Eq. (18)] has also been shown, for general spin. Border corrections to the field allow exact separability also in open chains.

We have then examined the magnetic behavior of a finite spin $1 / 2 X Y$ dimer chain. The factorizing field corresponds to the last parity transition exhibited by the exact GS for increasing field. Dimerization breakdown takes then place in steps, with all signatures of dimer structure being completely lost at the factorizing point: for a uniform field, the concurrence between any two spins approaches there (at each side) a constant value, independent of separation and coupling ratio $v^{e} / v^{o}$. The same behavior occurs in an alternating field, except that in this case there are three different concurrence side limits at separability, which depend on the odd-even field ratio. The entanglements between spins unconnected by the coupling may here exceed that between connected pairs. 
The previous properties are not a particular feature of the system considered. For full-range coupling, the same behavior is obtained at separability, as the eigenstates become there independent of the coupling range. The behavior of the concurrence between one spin and the rest of the chain has also been examined. An alternating field can induce opposite magnetizations at even and odd sites before the first transition, leading to a nonmonotonous behavior of this concurrence for increasing fields with two saturation points. The present results shed light on the complex behavior of entanglement in these systems and its relation with factorization. The exposed features can make such finite critical systems of special interest for diverse applications.

\section{ACKNOWLEDGMENTS}

The authors acknowledge support from CIC (R.R.) and CONICET (N.C. and J.M.M.) of Argentina.

\section{APPENDIX: EXACT SOLUTION OF THE DIMER CHAIN WITH ALTERNATING FIELD}

By means of the Jordan-Wigner transformation, ${ }^{35}$ and for a fixed value $p= \pm$ of the global $S_{z}$-parity $P_{z}$, we may exactly rewrite the dimer $X Y$ Hamiltonian (12) for $s_{\sigma}=\frac{1}{2}$ as a quadratic form in standard fermion creation and annihilation operators $c_{j}^{\dagger}$ and $c_{j}$. For an alternating field $b^{i}=b^{\sigma_{j}}$, with $\sigma_{j}$ $=o, e$ the site parity, we obtain in the cyclic case,

$$
H_{d}^{p}=\sum_{j=1}^{n}\left[b^{\sigma_{j}}\left(c_{j}^{\dagger} c_{j}-\frac{1}{2}\right)-\eta_{j}^{p}\left(v_{+}^{\sigma_{j}} c_{j}^{\dagger} c_{j+1}+v_{-}^{\sigma_{j}} c_{j}^{\dagger} c_{j+1}^{\dagger}+\text { h.c. }\right)\right],
$$

where $n+1 \equiv 1, v_{ \pm}^{\sigma}=\frac{1}{4}\left(v_{x}^{\sigma} \pm v_{y}^{\sigma}\right), \eta_{j}^{-}=1$, and $\eta_{j}^{+}=1-2 \delta_{j n}$. By means of separate discrete parity dependent Fourier transforms for even and odd sites,

$$
\left(\begin{array}{c}
c_{2 j-1}^{\dagger} \\
c_{2 j}^{\dagger}
\end{array}\right)=\frac{1}{\sqrt{n / 2}} \sum_{k \in k_{ \pm}} e^{-i \omega k j}\left(\begin{array}{c}
c_{k o}^{\prime \dagger} \\
c_{k e}^{\prime \dagger}
\end{array}\right), \quad \omega=\frac{4 \pi}{n},
$$

where $k_{+}=\left\{\frac{1}{2}, \ldots, \frac{n}{2}-\frac{1}{2}\right\}, k_{-}=\left\{0, \ldots, \frac{n}{2}-1\right\}$, we may rewrite (A1) as

$$
\begin{aligned}
H_{d}^{p} & =\sum_{k \in k_{p}}\left\{\sum_{\sigma} b^{\sigma} c_{k \sigma}^{\prime \dagger} c_{k \sigma}^{\prime}-\left[v_{+}^{k} c_{k o}^{\prime \dagger} c_{k e}^{\prime}+v_{-}^{k} c_{k o}^{\prime \dagger} c_{-k e}^{\prime \dagger}+\text { h.c. }\right]\right\} \\
& =\sum_{k \in k_{p}} \sum_{\nu= \pm} \lambda_{k}^{\nu}\left(a_{k \nu}^{\dagger} a_{k \nu}-\frac{1}{2}\right),
\end{aligned}
$$

where $v_{+}^{k}=v_{+}^{o} \pm v_{ \pm}^{e} e^{-i \omega k}$. The final diagonal form (A2) is obtained by means of a Bogoliubov transformation $c_{k \sigma}^{\prime \dagger}$ $=\sum_{\nu= \pm} U_{k \sigma}^{\nu} a_{k \nu}^{\dagger}+V_{k \sigma}^{\nu} a_{-k \nu}$ determined through the diagonalization of $4 \times 4$ blocks

$$
\mathcal{H}_{k}=\left(\begin{array}{cccc}
b^{o} & -v_{+}^{k} & 0 & -v_{-}^{k} \\
-\bar{v}_{+}^{k} & b^{e} & \bar{v}_{-}^{k} & 0 \\
0 & v_{-}^{k} & -b^{o} & v_{+}^{k} \\
-\bar{v}_{-}^{k} & 0 & \bar{v}_{+}^{k} & -b^{e}
\end{array}\right)
$$

whose eigenvalues are $\pm \lambda_{k}^{+}$and $\pm \lambda_{k}^{-}$, with

$$
\lambda_{k}^{ \pm 2}=\Delta \pm \sqrt{\Delta^{2}-\left|b^{o} b^{e}-\left(v_{+}^{k}+v_{-}^{k}\right)\left(\bar{v}_{+}^{k}-\bar{v}_{-}^{k}\right)\right|^{2}},
$$

and $\Delta=\frac{\left(b^{o}\right)^{2}+\left(b^{c}\right)^{2}}{2}+\left|v_{+}^{k}\right|^{2}+\left|v_{-}^{k}\right|^{2}$. Care should be taken to select the correct signs of $\lambda_{k}^{ \pm}$in order that the vacuum of the operators $a_{k \nu}$ has the proper $S_{z}$ parity and represents the lowest state for this parity.

The spin correlations in the lowest states for each $z$ parity can then be obtained from the ensuing basic fermionic contractions $f_{i j}=\left\langle c_{i}^{\dagger} c_{j}\right\rangle-\frac{1}{2} \delta_{i j}$ and $g_{i j}=\left\langle c_{i}^{\dagger} c_{j}^{\dagger}\right\rangle$, which can be directly obtained from the inverse Fourier transform of $\left\langle c_{k \sigma}^{\prime \dagger} c_{k \sigma^{\prime}}^{\prime}\right\rangle=\Sigma_{\nu} V_{k \sigma}^{\nu} \bar{V}_{k \sigma^{\prime}}^{\nu}$ and $\left\langle c_{k \sigma^{\prime}}^{\prime \dagger} c_{-k \sigma^{\prime}}^{\prime \dagger}\right\rangle=\Sigma_{\nu} V_{k \sigma}^{\nu} U_{-k \sigma^{\prime}}^{\nu}$. We then obtain, through the use of Wick's theorem, $\left\langle s_{i}^{z}\right\rangle=f_{i i},\left\langle s_{i}^{z} s_{j}^{z}\right\rangle$ $=f_{i i} f_{j j}-f_{i j}^{2}+g_{i j}^{2}$, and $\left\langle s_{i}^{+} s_{j}^{\mp}\right\rangle=\frac{1}{4}\left[\operatorname{det}\left(A_{i j}^{+}\right) \pm \operatorname{det}\left(A_{i j}^{-}\right)\right]$, where $A_{i j}^{ \pm}$ are $(i-j) \times(i-j)$ matrices of elements $2(f+g)_{i+p+{ }_{1}^{0}, i+q+1}$.
${ }^{1}$ C. H. Bennett, G. Brassard, C. Crépeau, R. Jozsa, A. Peres, and W. K. Wootters, Phys. Rev. Lett. 70, 1895 (1993); C. H. Bennett, G. Brassard, S. Popescu, B. Schumacher, J. A. Smolin, and W. K. Wootters, ibid. 76, 722 (1996).

${ }^{2}$ A. K. Ekert, Phys. Rev. Lett. 67, 661 (1991); Nature (London) 358, 14 (1992)

${ }^{3}$ G. Burkard, D. Loss, and D. P. DiVincenzo, Phys. Rev. B 59, 2070 (1999); D. P. DiVincenzo, D. Bacon, J. Kempe, G. Burkard, and K. B. Whaley, Nature (London) 408, 339 (2000).

${ }^{4}$ M. A. Nielsen and I. Chuang, Quantum Computation and Quantum Information (Cambridge University Press, Cambridge, 2000).

${ }^{5}$ L. Amico, R. Fazio, A. Osterloh, and V. Vedral, Rev. Mod. Phys. 80, 517 (2008).

${ }^{6}$ T. J. Osborne and M. A. Nielsen, Phys. Rev. A 66, 032110 (2002).

${ }^{7}$ G. Vidal, J. I. Latorre, E. Rico, and A. Kitaev, Phys. Rev. Lett. 90, 227902 (2003)
${ }^{8}$ L. M. Duan, E. Demler, and M. D. Lukin, Phys. Rev. Lett. 91, 090402 (2003); M. J. Hartmann, F. G. S. L. Brandao, and M. B. Plenio, ibid. 99, 160501 (2007).

${ }^{9}$ J. Cho, D. G. Angelakis, and S. Bose, Phys. Rev. A 78, 062338 (2008).

${ }^{10}$ J. Kurmann, H. Thomas, and G. Müller, Physica A 112, 235 (1982).

${ }^{11}$ G. Müller and R. E. Shrock, Phys. Rev. B 32, 5845 (1985).

${ }^{12}$ T. Roscilde, P. Verrucchi, A. Fubini, S. Haas, and V. Tognetti, Phys. Rev. Lett. 93, 167203 (2004); 94, 147208 (2005).

${ }^{13}$ S. Dusuel and J. Vidal, Phys. Rev. B 71, 224420 (2005).

${ }^{14}$ L. Amico, F. Baroni, A. Fubini, D. Patanè, V. Tognetti, and P. Verrucchi, Phys. Rev. A 74, 022322 (2006); F. Baroni, A. Fubini, V. Tognetti, and P. Verrucchi, J. Phys. A 40, 9845 (2007).

${ }^{15}$ S. M. Giampaolo and F. Illuminati, Phys. Rev. A 76, 042301 (2007); S. M. Giampaolo, F. Illuminati, P. Verrucchi, and S. De Siena, ibid. 77, 012319 (2008).

${ }^{16}$ R. Rossignoli, N. Canosa, and J. M. Matera, Phys. Rev. A 77, 
052322 (2008).

${ }^{17}$ S. M. Giampaolo, G. Adesso, and F. Illuminati, Phys. Rev. Lett. 100, 197201 (2008); Phys. Rev. B 79, 224434 (2009).

${ }^{18}$ S. M. Giampaolo, G. Adesso, and F. Illuminati, arXiv:0906.4451 (unpublished).

${ }^{19}$ G. L. Giorgi, Phys. Rev. B 79, 060405(R) (2009); 80, 019901(E) (2009).

${ }^{20}$ R. Rossignoli, N. Canosa, and J. M. Matera, Phys. Rev. A 80, 062325 (2009).

${ }^{21}$ D. Kaszlikowski, W. Son, and V. Vedral, Phys. Rev. A 76, 054302 (2007).

${ }^{22}$ M.-G. Hu, K. Xue, and M.-L. Ge, Phys. Rev. A 78, 052324 (2008).

${ }^{23}$ F. T. Arecchi, E. Courtens, R. Gilmore, and H. Thomas, Phys. Rev. A 6, 2211 (1972).

${ }^{24} \mathrm{P}$. Ring and P. Schuck, The Nuclear Many Body Problem (Springer, New York, 1980).

${ }^{25}$ J. M. Matera, R. Rossignoli, and N. Canosa, Phys. Rev. A 78, 012316 (2008).
${ }^{26}$ N. Canosa and R. Rossignoli, Phys. Rev. A 75, 032350 (2007).

${ }^{27}$ S. Hill and W. K. Wootters, Phys. Rev. Lett. 78, 5022 (1997); W. K. Wootters, ibid. 80, 2245 (1998).

${ }^{28}$ P. Rungta, V. Buzek, C. M. Caves, M. Hillery, and G. J. Milburn, Phys. Rev. A 64, 042315 (2001).

${ }^{29}$ P. Rungta and C. M. Caves, Phys. Rev. A 67, 012307 (2003).

${ }^{30}$ V. Coffman, J. Kundu, and W. K. Wootters, Phys. Rev. A 61, 052306 (2000).

${ }^{31}$ T. J. Osborne and F. Verstraete, Phys. Rev. Lett. 96, 220503 (2006).

${ }^{32}$ H. J. Lipkin, N. Meshkov, and A. J. Glick, Nucl. Phys. 62, 188 (1965)

${ }^{33}$ M. Koashi, V. Buzek, and N. Imoto, Phys. Rev. A 62, 050302(R) (2000); W. Dür, ibid. 63, 020303 (2001).

${ }^{34}$ W. Dür, G. Vidal, and J. I. Cirac, Phys. Rev. A 62, 062314 (2000).

${ }^{35}$ E. Lieb, T. Schultz, and D. Mattis, Ann. Phys. (N.Y.) 16, 407 (1961). 